\title{
Binaural Integrated Active Noise Control and Noise Reduction in Hearing Aids
}

\author{
Serizel, Romain; Moonen, Marc; Wouters, Jan; Jensen, Søren Holdt
}

Published in:

I E E E Transactions on Audio, Speech and Language Processing

Link to article, DOI:

10.1109/TASL.2012.2234111

Publication date:

2013

Document Version

Publisher's PDF, also known as Version of record

Link back to DTU Orbit

Citation $(A P A)$ :

Serizel, R., Moonen, M., Wouters, J., \& Jensen, S. H. (2013). Binaural Integrated Active Noise Control and Noise Reduction in Hearing Aids. I E E E Transactions on Audio, Speech and Language Processing, 21(5), 1113-1118. https://doi.org/10.1109/TASL.2012.2234111

\section{General rights}

Copyright and moral rights for the publications made accessible in the public portal are retained by the authors and/or other copyright owners and it is a condition of accessing publications that users recognise and abide by the legal requirements associated with these rights.

- Users may download and print one copy of any publication from the public portal for the purpose of private study or research.

- You may not further distribute the material or use it for any profit-making activity or commercial gain

- You may freely distribute the URL identifying the publication in the public portal 


\section{Binaural Integrated Active Noise Control and Noise Reduction in Hearing Aids}

\author{
Romain Serizel, Marc Moonen, Jan Wouters, and \\ Søren Holdt Jensen
}

\begin{abstract}
This paper presents a binaural approach to integrated active noise control and noise reduction in hearing aids and aims at demonstrating that a binaural setup indeed provides significant advantages in terms of the number of noise sources that can be compensated for and in terms of the causality margins.
\end{abstract}

Index Terms-Hearing aids, noise reduction, multichannel Wiener filter, active noise control, binaural processing.

\section{INTRODUCTION}

Binaural hearing offers advantages over monaural hearing such as a better speech intelligibility, enhanced localization, improved quality of listening [1]-[3]. If binaural information is really helpful for normal hearing persons, it may become tremendously important for persons with a hearing impairment.

State-of-the-art hearing aids perform noise reduction (NR) in order to improve their output signal-to-noise ratio (SNR) and hence to allow for a better speech understanding in background noise and to ease listening effort [4]. Conventional NR systems such as the generalized sidelobe canceller (GSC) [5] or techniques based on the multichannel Wiener filter (MWF) [6], [7] can be used.

When these processing schemes are applied in a monaural setup or a bilateral setup (i.e., a setup with two hearing aids working independently), the SNR improvement can come with a degradation of binaural localization cues, which can put the hearing aid user at a disadvantage. In a binaural setup, two hearing aids are worn, which can communicate each other, e.g., via a wireless link. The NR schemes applied in hearing aids can be adapted to take advantage of this setup to deliver improved SNR [8] and to preserve binaural localization cues [9].

Hearing aids with an open fitting (i.e., where the earmold is replaced by a simple tube) can improve the physical comfort [10] and have become more common over the past years. Moreover, conventional NR techniques using monaural, bilateral or binaural processing do not

Manuscript received February 27, 2012; revised September 19, 2012, November 20, 2012; accepted November 23, 2012. Date of publication December 20, 2012; date of current version February 25, 2013. This research work was carried out at the ESAT Laboratory of Katholieke Universiteit Leuven, in the frame of K.U. Leuven Research Council CoE EF/05/006 Optimization in Engineering (OPTEC), PFV/10/002 (OPTEC), Concerted Research Action GOA-MaNet, the Belgian Program on Interuniversity Attraction Poles initiated by the Belgian Federal Science Policy Office IUAP P6/04 (DYSCO, "Dynamical systems, control and optimization," 2007-2011), Research Project FWO nr. G.0600.08 ("Signal processing and network design for wireless acoustic sensor networks"), EC-FP6 project SIGNAL: "Core Signal Processing Training Program," The scientific responsibility is assumed by its authors. The associate editor coordinating the review of this manuscript and approving it for publication was Dr. Man-Wai Mak.

R. Serizel and M. Moonen are with the Department of Electrical Engineering, Katholieke Universiteit Leuven, ESAT-SCD, B-3001 Leuven, Belgium, and also with the IBBT Future Health Department, Leuven, Belgium (e-mail: romain.serizel@esat.kuleuven.be; marc.moonen@esat.kuleuven.be).

$\mathrm{J}$. Wouters is with the Department of Neuroscience, Katholieke Universiteit Leuven, B-3000 Leuven, Belgium (e-mail: jan.wouters@med.kuleuven.be).

S. H. Jensen is with the Department of Electronic Systems, Aalborg University, Niels DK-9220 Aalborg, Denmark (e-mail: shj@es.aau.dk).

Color versions of one or more of the figures in this paper are available online at http://ieeexplore.ieee.org.

Digital Object Identifier 10.1109/TASL.2012.2234111 take leakage effects into account, which can be significant whenever an open fitting is used. Combined with the attenuation in the secondary path, i.e., the acoustic path from the hearing aid loudspeaker to the eardrum, the noise leaking through the open fitting directly to the eardrum can then override the action of the NR. One efficient way to tackle this problem is to use an active noise control (ANC) [10], [11] combined with the NR. In [12], an MWF-based monaural integrated ANC and NR has been introduced.

To be effective, the integrated ANC and NR scheme needs to be designed as a causal system. In a monaural setup, the causality margins depend on the distance between the hearing aid microphones and the ear canal. These margins are therefore rather small and the causality may quickly become a limitation [12]. It has also been shown in [13] that in a single speech source scenario, the integrated ANC and NR scheme can compensate for noise sources only as long as the number of sources (speech source and noise sources) is less than or equal to the number of microphones. In a monaural setup the number of sources that can be compensated for is therefore limited by the number of microphones on one hearing aid (which is maximally three in the case of commercial hearing aids).

In this paper, the monaural integrated $\mathrm{ANC}$ and $\mathrm{NR}$ scheme presented in [12] is extended to a binaural setup. It is then investigated how a binaural integrated ANC and NR scheme can benefit from the causality margin increase owing to the (outpost) location of the contralateral microphones. The binaural integrated ANC and NR is also applied to a multiple noise sources scenario in order to confirm the analysis conducted in [13] on the number of sources that can be compensated for and to confirm the benefits from the increased number of available microphones.

The signal model, the binaural MWF-based NR and ANC, the secondary path and the signal leakage problem as well as the causality issues in ANC schemes are described in Section II. The binaural MWFbased integrated ANC and NR scheme is presented in Section III. Experimental results are presented in Section IV and finally conclusions are presented in Section V.

\section{BACKGround AND PROBlem Statement}

This section introduces the signal model and notation, the binaural MWF-based NR and ANC, the secondary path and signal leakage problems as well as the causality issues in ANC schemes.

\section{A. Signal Model}

In an ideal binaural setup, microphone signals from both hearing aids are used to compute the hearing aid loudspeaker signals. Let $M$ be the number of microphones (channels) on each hearing aid and $N$ the NR and/or ANC filter length. The time-domain signal $x_{L, m}[k]$ for microphone $m$ in the left hearing aid has a desired speech part $x_{L, m}^{s}[k]$ and an additive noise part $x_{I_{,}, m}^{n}[k]$ :

$$
x_{L, m}[k]=x_{L, m}^{s}[k]+x_{L, m}^{n}[k] \quad m \in\{1 \ldots M\}
$$

where $k$ is the time index.

In practice, in order to distinguish "speech plus noise periods" from "noise only periods" it is necessary to use a voice activity detector (VAD). The performance of the VAD can affect the performance of the filters. In this paper however, in order to exclude VAD error effects from the analysis, a perfect VAD is assumed.

The column vector $\mathbf{x}_{L, m}[k]$ contains the $N$ most recent samples of the microphone signal $m$ in the left hearing aid:

$$
\mathbf{x}_{L, m}[k]=\left[x_{L, m}[k] \ldots x_{L, m}[k-N+1]\right]^{T} \quad m \in\{1 \ldots M\}
$$


The time-domain signal $x_{R, m}[k]$ and vector $\mathbf{x}_{R, m}[k]$ for microphone $m$ in the right hearing aid can be specified similarly.

The $M N$-dimensional compound vectors $\mathbf{x}_{L}[k]$ and $\mathbf{x}_{R}[k]$ gathering all microphone signals from the left and the right hearing aid respectively and the $2 M N$-dimensional compound signal vector $\mathbf{x}[k]$ are defined as follows:

$$
\begin{aligned}
\mathbf{x}_{L}[k] & =\left[\mathbf{x}_{L, 1}[k]^{T} \ldots \mathbf{x}_{L, M}[k]^{T}\right]^{T} \\
\mathbf{x}_{R}[k] & =\left[\mathbf{x}_{R, 1}[k]^{T} \ldots \mathbf{x}_{R, M}[k]^{T}\right]^{T} \\
\mathbf{x}[k] & =\left[\mathbf{x}_{L}[k]^{T} \mathbf{x}_{R}[k]^{T}\right]^{T}
\end{aligned}
$$

\section{B. Binaural MWF-Based NR, Secondary Path and Signal Leakage}

In the subsequent sections, only the filter designed for the left hearing aid $\left(\mathbf{w}_{L}[k]\right)$ will be considered. All the derivations, however, also hold for the filter in the right hearing aid $\left(\mathbf{w}_{R}[k]\right)$. For conciseness, the filter $\mathbf{w}_{L}[k]$ will be denoted as $\mathbf{w}[k]$ in the remainder of the paper.

The binaural MWF-based NR filter $\mathbf{w}_{\mathrm{NR}}[k]$ is designed to minimize the following mean squared error (MSE) criterion:

$$
\begin{aligned}
J_{\mathrm{NR}}(\mathbf{w}[k]) & =\mathbb{E}\left\{\left|\mathbf{w}[k]^{T} \mathbf{x}[k]-G \cdot x_{L, 1}^{s}[k-\Delta]\right|^{2}\right\} \\
& =\mathbb{E}\left\{\left|\mathbf{w}[k]^{T} \mathbf{x}[k]-d_{\mathrm{NR}, L}[k]\right|^{2}\right\}
\end{aligned}
$$

where $\mathbb{E}\{\cdot\}$ is the expectation operator, $G$ the desired gain and $d_{\mathrm{NR}, L}[k]$ is the desired signal for the NR. The delay $\Delta$ is a design parameter. The speech part in the first microphone is used here as the desired signal for the Wiener filter.

The MWF minimizing (6) is then:

$$
\mathbf{w}_{\mathrm{NR}}[k]=\mathbf{R}_{x}[k]^{-1} \mathbf{r}_{x d_{N R}}[k]
$$

where $\mathbf{R}_{x}[k]$ is the correlation matrix of the microphone signals $\mathbf{x}[k]$ and $\mathbf{r}_{x d_{N R}}[k]$ is the cross-correlation vector between the input $\mathbf{x}[k]$ and the desired signal $d_{\mathrm{NR}, L}[k]$. The correlation matrix $\mathbf{R}_{x}[k]$ is assumed to have full rank. The estimation of $\mathbf{r}_{x d_{N R}}[k]$ relies on a voice activity detection [7]

The NR output signal is then:

$$
z[k]=\mathbf{w}_{\mathrm{NR}}[k]^{T} \mathbf{x}[k]
$$

The conventional NR filters are designed without taking the effect of the signal leakage and the secondary path effect into account. The secondary path represents the propagation from the loudspeaker to the eardrum and usually acts as an attenuation [12]. Assuming that the loudspeaker characteristic is approximately linear, the secondary path can be represented by a filter coefficient vector $\mathbf{c}[k]$ of length $P$. A hearing aid with an open fitting has no earmold to prevent ambient sound from leaking into the ear canal, which results in additional leakage signal $l[k]$ reaching the eardrum.

Taking both the signal leakage and the secondary path effect into account, leads to the following model for the eardrum signal:

$$
\tilde{z}[k]=\mathbf{c}[k]^{T}[z[k] \ldots z[k-P+1]]^{T}+l[k]
$$

It clearly appears that for small gains $G$ the leakage SNR may affect the output SNR thus partly cancelling the improvement achieved with the NR. A feedforward ANC strategy can then be applied to compensate for the degradation introduced by the noise leakage.

\section{Binaural MWF-Based ANC and Causality}

The goal of the binaural ANC is to extend the monaural ANC based on existing work on binaural MWF-based NR [9], [14]. The binaural signal model from [9], [14] is applied to the ANC case to define a binaural ANC error criterion. In this paper, it is assumed that a microphone is present in the ear canal to provide the signal $\tilde{z}[k]$. Commercial hearing aids currently do not have an ear canal microphone, but it is technically possible to include a microphone at the end of the tube that is used to conduct the sound from the BTE to the ear canal, which is then sufficiently close to the eardrum.

The binaural ANC scheme relies on a Filtered-x structure. The filtered reference signal is defined as:

$$
\mathbf{y}[k]=\left[\mathbf{y}_{L}[k]^{T} \mathbf{y}_{R}[k]^{T}\right]^{T}
$$

where $\mathbf{y}_{L}[k]$ is the filtered reference signal derived from the microphone signals in the left hearing aid:

$$
\begin{aligned}
y_{L, m}[k] & =\hat{\mathbf{c}}[k]^{T}\left[x_{L, m}[k] \ldots x_{L, m}[k-\hat{P}+1]\right]^{T} \\
\mathbf{y}_{L, m}[k] & =\left[y_{L, m}[k] \ldots y_{L, m}[k-N+1]\right]^{T} m \in\{1 \ldots M\} \\
\mathbf{y}_{L}[k] & =\left[\mathbf{y}_{L, 1}[k]^{T} \ldots \mathbf{y}_{L, M}[k]^{T}\right]^{T}
\end{aligned}
$$

with $\hat{\mathbf{c}}[k]$ a model of $\mathbf{c}[k]$ and where $\mathbf{y}_{R}[k]$, the filtered reference signal derived from the microphone signals in the right hearing aid, is similarly defined.

The binaural MWF-based ANC filter $\mathbf{w}_{\mathrm{ANC}}[k]$ is designed to minimize the MSE:

$$
J_{\mathrm{ANC}}(\mathbf{w}[k])=\mathbb{E}\left\{\left|\mathbf{c}[k]^{T}[z[k] \ldots z[k-P+1]]^{T}+l[k]\right|^{2}\right\}
$$

where $z[k]$ is the output signal of the filter $\mathbf{w}[k]$. Assuming that the secondary path identification error is small $(\hat{\mathbf{c}}[k] \approx \mathbf{c}[k]$ and $\left.y[k] \approx \mathbf{c}[k]^{T}\left[x_{L, m}[k] \ldots x_{L_{,}, m}[k-P+1]\right]^{T}\right)$ and that the filter $\mathbf{w}[k]$ is adapting slowly (so that $\mathbf{w}[k]$ and $\mathbf{c}[k]$ can be interchanged), the MSE criterion (13) can be written as follows:

$$
J_{\mathrm{ANC}}(\mathbf{w}[k]) \approx \mathbb{E}\left\{\left|\mathbf{w}[k]^{T} \mathbf{y}[k]+l[k]\right|^{2}\right\}
$$

The Filtered-x MWF (FxMWF) minimizing (14) is then:

$$
\mathbf{w}_{\mathrm{ANC}}[k]=-\mathbf{R}_{y}[k]^{-1} \mathbf{r}_{y l}[k]
$$

where $\mathbf{R}_{y}[k]$ is the correlation matrix of the filtered microphone signals $\mathbf{y}[k]$ and $\mathbf{r}_{y l}[k]$ is the cross-correlation vector between the filtered microphone signals $\mathbf{y}[k]$ and the leakage signal $l[k]$. The correlation matrix $\mathbf{R}_{y}[k]$ is assumed to have full rank. In practice, the leakage signal $l[k]$ can be estimated from the ear canal microphone signal $\tilde{z}[k]$ and the loudspeaker signal $z[k]$ :

$$
l[k] \approx \tilde{z}[k]-\hat{\mathbf{c}}[k]^{T}[z[k] \ldots z[k-P+1]]^{T}
$$

The performance of a feedforward ANC scheme is highly dependent on the causality of the system [10]. The distance between the hearing aid microphones and the hearing aid loudspeaker must be sufficient to allow a causal design. In particular, the acoustic delay (i.e., direct propagation) from the noise source to the ear canal microphone $\Delta_{\text {pri }}$ has to be larger than the sum of the acoustic delay from the noise source to one of the hearing aid microphones $\Delta_{\text {ref }}$, the delay associated with the processing within the hearing aid $\delta$ (i.e., Analogue-to-Digital (A/D) converter delays, Digital-to-Analogue (D/A) converter delays, wireless link delays...), and the acoustic delay of the secondary path $\Delta_{\text {sec }}$ (Fig. 1).

Here, $\delta$ is used as a parameter to determine how much delay the system can add in the signal path before the ANC performance starts to decrease. The limit value for $\delta$ will be referred to as the causality 


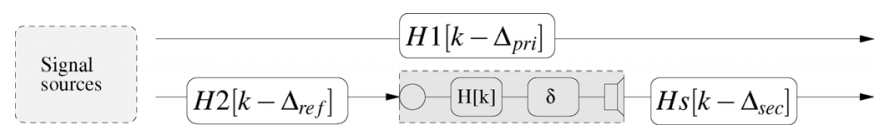

Fig. 1. Delays in hearing aid system.

margin $\delta_{0}=\Delta_{\text {pri }}-\Delta_{\text {ref }}-\Delta_{\text {sec }}$. When the causality margin $\delta_{0}$ is positive, it is possible to have delays in the signal path, and so the system is said to be causal:

$$
\Delta_{\text {pri }} \geq \Delta_{\text {ref }}+\Delta_{\text {sec }}
$$

When the causality margin $\delta_{0}$ is negative, the ANC has to act as a linear predictor. The system is then said to be non-causal:

$$
\Delta_{\text {pri }}<\Delta_{\text {ref }}+\Delta_{\text {sec }}
$$

In practice, this criterion does not define a hard limit but it gives an indication on the performance to be expected from an ANC scheme [15].

\section{BINAURAL MWF-BASED INTEGRATED ANC AND NR}

The goal of the binaural MWF-based integrated ANC and NR is to extend the MWF-based integrated ANC and NR scheme presented in [12]. The binaural integrated ANC and NR can then benefit from the extra contra-lateral hearing aid microphones in order to compensate for the effect of more noise sources. The ANC part of the integrated ANC and NR scheme can also benefit from the causality margin improvement owing to the contra-lateral hearing aid microphones location.

As speech component of the leakage can actually be helpful, e.g., for localisation, it is chosen here to cancel only the noise component of the leakage. The overall desired signal (at the eardrum) to be used is then:

$$
d_{\text {Int }, L}[k]=d_{\mathrm{NR}, L}[k]+l^{s}[k]
$$

where $d_{\mathrm{NR}, L_{.}}[k]$ is defined in (6).

Hence the MSE criterion to be minimized is:

$$
\begin{aligned}
J_{\text {Int }}(\mathbf{w}[k])=\mathbb{E}\left\{\mid \mathbf{c}[k]^{T}[z[k] \ldots z[k-P+1]]^{T}\right. \\
+\underbrace{l[k]-l^{s}[k]}_{l^{n}[k]}-\left.d_{\mathrm{NR}, L}[k]\right|^{2}\}
\end{aligned}
$$

where $z[k]$ is the output signal of the binaural filter $\mathbf{w}[k]$. The MSE criterion (20) is then the same as the MSE criterion introduced in [12] for the monaural integrated ANC and NR except that the filter $\mathbf{w}[k]$ is now applied to a binaural input.

Assuming that the secondary path identification error is small $(\hat{\mathbf{c}}[k] \approx \mathbf{c}[k])$ and that the filter $\mathbf{w}[k]$ is adapting slowly, the MSE criterion (20) can be written as follows:

$$
J_{\mathrm{Int}}[k] \approx \mathbb{E}\left\{\left|\mathbf{w}[k]^{T} \mathbf{y}[k]+l^{n}[k]-d_{\mathrm{NR}, L}[k]\right|^{2}\right\}
$$

Assuming that speech and noise are uncorrelated, the FxMWF minimizing (20) is then:

$$
\mathbf{w}_{\text {Int }}[k]=\mathbf{R}_{y}^{-1}[k]\left(\mathbf{r}_{y^{s} d_{\mathrm{NR}, L}}[k]-\mathbf{r}_{y^{n} l^{n}}[k]\right)
$$

Here $\mathbf{R}_{y}[k]$ is the correlation matrix of the filtered microphone signals $\mathbf{y}[k]$ and $\mathbf{r}_{y}{ }^{s} d_{\mathrm{NR}, L}[k]$ is the cross-correlation vector between the speech component of the filtered microphone signals $\mathbf{y}^{s}[k]$ and the desired signal $d_{\mathrm{NR}, L}[k]$ which can again be estimated based on a voice

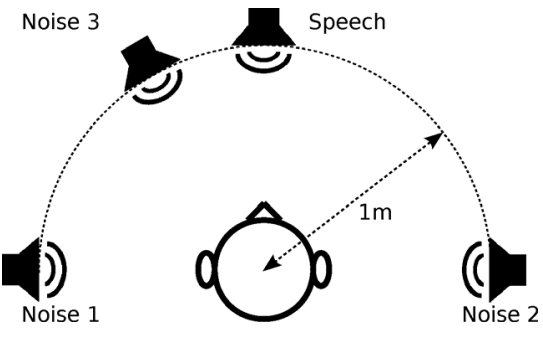

Fig. 2. Experimental setup.

activity detection. The correlation matrix $\mathbf{R}_{y}[k]$ is assumed to have full rank. Finally $\mathbf{r}_{y^{n} l^{n}}[k]$ is the cross-correlation vector between the noise component of the filtered microphone signals $\mathbf{y}^{n}[k]$ and the noise component of the leakage signal $l^{n}[k]$. The noise component of the leakage can be estimated similarly as in (16) during noise only periods. A description of how these statistics are computed in practice can be found in [12] (Section III-D).

\section{EXPERIMENTAL RESULTS}

\section{A. Experimental Setup}

The simulations were done with acoustic paths measured on a CORTEX MK2 manikin head and torso equipped with artificial ears and two-microphones behind-the-ear (BTE) hearing aids. The sound sources (FOSTEX 6301B loudspeakers) were positioned at 1 meter from the centre of the head. The speech source was located at $0^{\circ}$ and the noise sources at $90^{\circ}, 270^{\circ}$ and $330^{\circ}$ (see Fig. 2). The hearing aid considered here is the left hearing aid, facing the noise source at $270^{\circ}$. Commercial hearing aids currently do not have an ear canal microphone, therefore the artificial ear eardrum microphone is used here to generate the ear canal microphone signal. The tests were run on 22 seconds long signals. The speech was composed of three sentences from the HINT database [16] concatenated with silence periods. The noise was the multitalker babble from Auditec [17]. All signals were sampled at $16 \mathrm{kHz}$.

The filter length is set to $N=128$, and the NR delay is set to half the NR filter length $(\Delta=64)$. If the speech component and the noise component of the microphone signals are assumed to be uncorrelated, it has been shown in [12] that the integrated ANC and NR can be decomposed into the sum of two sets of filters, one for NR and the other for the ANC. The NR delay $(\Delta)$ then does not affect the causality margin of the ANC part of the integrated ANC and NR scheme. The secondary path $\mathbf{c}[k]$ is estimated off-line using an identification technique based on the Normalized Least Mean Squares (NLMS) algorithm. The length of the estimated path $\hat{\mathbf{c}}[k]$ is set to $L=32$.

The performance measure used for the ANC schemes is the residual noise power improvement at the eardrum:

$$
\Delta \operatorname{Pow}^{n}[k]=\operatorname{Pow}_{\text {out }}^{n}[k]-\operatorname{Pow}_{\text {leak }}^{n}[k]
$$

where Pow ${ }_{\text {out }}^{n}[k]$ and $\operatorname{Pow}_{\text {leak }}^{n}[k]$ are the power (in $\mathrm{dB}$ ) of the residual noise and the power of the noise component of the leakage signal at the eardrum.

The performance measure used for integrated ANC and NR schemes is the intelligibility-weighted SNR [18] improvement where the leakage signal SNR is taken as a reference:

$$
\Delta \mathrm{SNR}_{\text {intell }}=\sum_{i} I_{i}\left(\mathrm{SNR}_{\mathrm{i}, \text { Int }}-\mathrm{SNR}_{\mathrm{i}, \text { leak }}\right)
$$

where $I_{i}$ is the band importance function defined in [19] and $\mathrm{SNR}_{\mathbf{i}, \mathrm{Int}}$ and $\mathrm{SNR}_{\mathrm{i}, \text { leak }}$ represent the output $\mathrm{SNR}$ of the integrated $\mathrm{ANC}$ and $\mathrm{NR}$ scheme and the leakage SNR (in $\mathrm{dB}$ ) of the $i$ th band, respectively. 


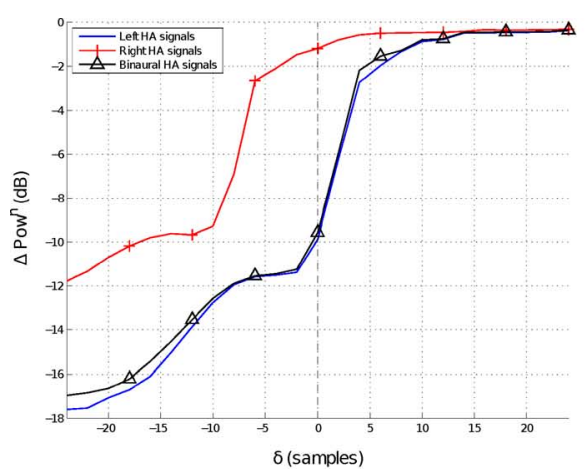

(a)

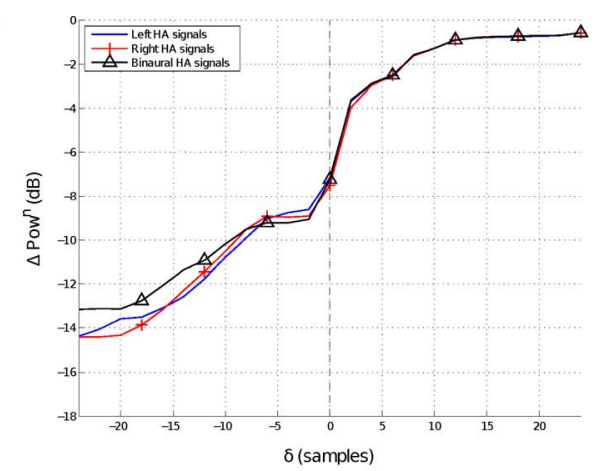

(b)

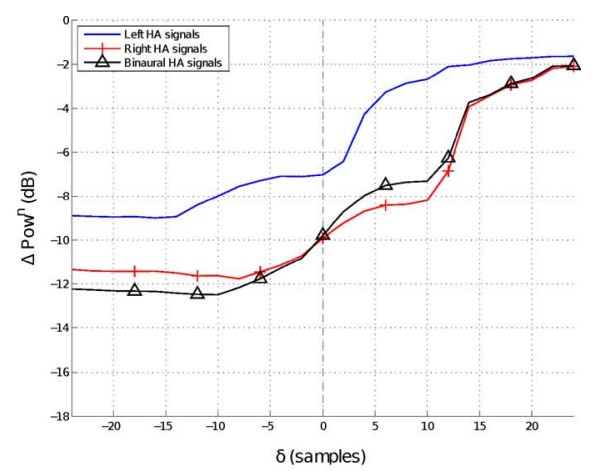

(c)

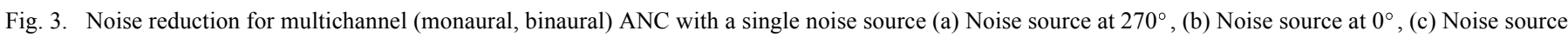
at $90^{\circ}$.

\section{B. Binaural ANC}

Three different 2-channel ANC schemes are compared. Two monaural schemes are considered (one using the microphone signals from the left hearing aid, the other one using the microphone signals from the right hearing aid) and compared to a binaural scheme using one microphone signal from each hearing aid. The binaural scheme can also run with four microphone signals, but only two microphone signals are used here in order to have a fair comparison with the monaural schemes.

1) Single Noise Source: The first experiment is set up with only one noise source. The noise source can be located at $0^{\circ}, 90^{\circ}$ or at $270^{\circ}$. In each case the three different schemes are run for different delay $\delta$ and the residual noise power performance is evaluated. In practice, the microphone signals are artificially delayed by a delay $\delta$.

Fig. 3(a) presents the residual noise power improvement at the left eardrum for the three schemes when the source is facing the left hearing aid $\left(270^{\circ}\right)$. The noise signal then reaches the microphones of the left hearing aid before reaching the left eardrum. It is therefore possible to design a causal system based on the microphone signals from the left hearing aid, even if the causality margin is rather small $\left(\delta_{0} \approx 2\right)$. On the other hand, the noise signal reaches the microphones of the right hearing aid after reaching the left eardrum. The ANC scheme using the right hearing aid microphone signals is then non-causal $\left(\delta_{0} \approx-8\right)$. The binaural ANC scheme is based on a microphone signal from each hearing aid and matches the residual noise power performance of the monaural scheme using the microphone signals from the left hearing aid.

The residual noise power improvement at the left eardrum when the noise source is facing the listener $\left(0^{\circ}\right)$ is shown on Fig. 3(b). The noise signal reaches the microphones of the left hearing aid at the same time as it reaches the microphones of the right hearing aid. The causality margins are then the same if the system is based on the microphone signals from the left hearing aid or from the right hearing aid $\left(\delta_{0} \approx 1\right)$. The binaural ANC scheme performance is, in this scenario, similar to the performance of the monaural schemes.

Fig. 3(c) presents the residual noise power improvement at the left eardrum for the three schemes when the noise source is facing the right hearing aid $\left(90^{\circ}\right)$. The noise signal then reaches the left eardrum shortly after it reaches the microphones of the left hearing aid. The monaural ANC scheme using the microphone signals from the left hearing aid then has to be designed with low causality margin $\left(\delta_{0} \approx 3\right)$. In this scenario however, the noise signal reaches the microphones of the right hearing aid before reaching the left eardrum. Therefore, the ANC scheme using the microphone signals from the right hearing aid can be designed with a larger causality margin $\left(\delta_{0} \approx 13\right)$. Once again

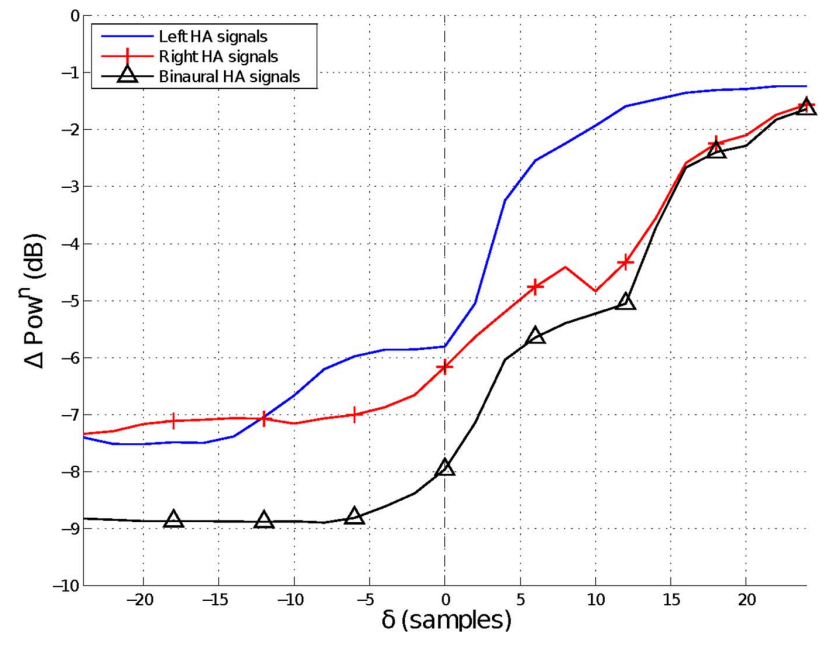

Fig. 4. Noise reduction for multichannel (monaural, binaural) active noise control with two noise sources $\left(270^{\circ}\right.$ and $\left.90^{\circ}\right)$.

the binaural ANC scheme matches the residual noise power performance of the best of the two monaural schemes.

2) Multiple Noise Sources: The second experiment is set up with two noise sources, one on each side of the head, i.e., one noise source at $270^{\circ}$ and the other noise source at $90^{\circ}$. The residual noise power improvement at the left eardrum is presented in Fig. 4. Each of the monaural ANC schemes is well suited to attenuate the noise signal from one of the sources but the attenuation of the other noise source can be problematic (see also above). The binaural ANC scheme on the other hand delivers a better performance than any of the monaural schemes in this particular case.

\section{Binaural Integrated $A N C$ and $N R$}

In this part, the performance of the integrated ANC and NR scheme is evaluated. The first experiment aims at showing the effect of causality on different integrated ANC and NR schemes while the second experiment focuses on the impact of the number of sources on the integrated ANC and NR schemes.

1) Single Noise Source: In the first experiment there is only one noise source which can be located at $90^{\circ}$ or at $270^{\circ}$. The gain $G$ is set to $10 \mathrm{~dB}$. For each scenario different schemes are run for different delay $\delta$ and their speech-intelligibility weighted SNR improvement is evaluated.

Three different 2-channel integrated ANC and NR schemes are compared here. As in the previous part, two monaural schemes are considered (one using the microphones signals from the left hearing aid, the 


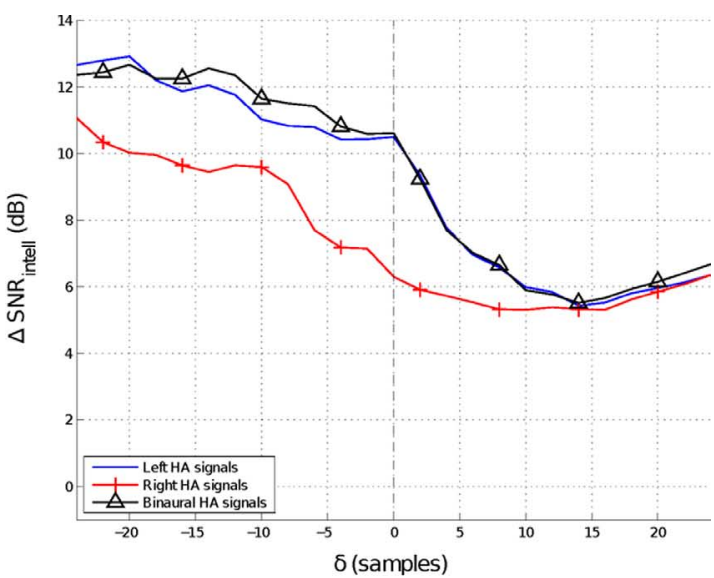

(a)

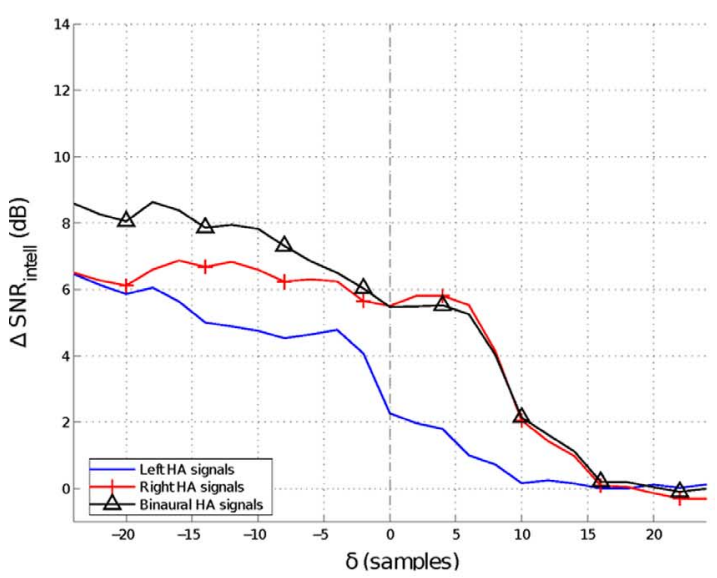

(b)

Fig. 5. Integrated active noise control and noise reduction for multichannel (monaural, binaural) active noise control with a single noise source. (a) Noise source at $\left(270^{\circ}\right),(b)$ noise source at $\left(90^{\circ}\right)$.

other one using the microphone signals from the right hearing aid) and compared to a binaural scheme using one microphone signal from each hearing aid.

Fig. 5(a) presents the SNR improvement at the left eardrum for the three schemes when the source is facing the left hearing aid $\left(270^{\circ}\right)$. In this scenario, a system based on the microphone signals from the left hearing aid would be causal and achieve an SNR improvement up to $\delta \approx 1$. A system based on the microphone signals from the right hearing aid on the other hand would be non-causal $\left(\delta_{0} \approx-8\right)$. The binaural scheme achieves a performance similar to the scheme based on the microphone signals from the left hearing aid and can achieve an SNR improvement up to $\delta \approx 1$.

The SNR power improvement at the left eardrum when the noise source is facing the right ear $\left(90^{\circ}\right)$ is shown in Fig. 5(b). In this scenario the integrated ANC and NR scheme based on the microphone signals from the left hearing aid has a negative causality margin $\left(\delta_{0} \approx-1\right)$ whereas the schemes based on the microphone signals from the right hearing aid delivers SNR improvement up to $\delta \approx 8$. Once again, the binaural scheme matches the SNR improvement performance of the best of the two monaural schemes.

2) Multiple Noise Sources: The aim of the second experiment is to evaluate the effect of the number of sound sources (speech source plus noise sources) on the performance of the integrated ANC and NR scheme. The causality margin of the system is (artificially) set to a positive value $\left(\delta_{0}=12\right)$. This value is chosen to be larger than the causality margin resulting from the propagation from the contra-lateral ear to the

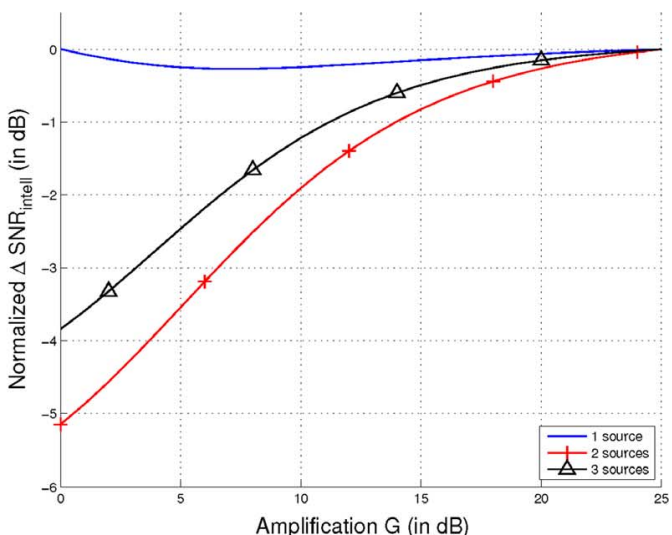

(a)

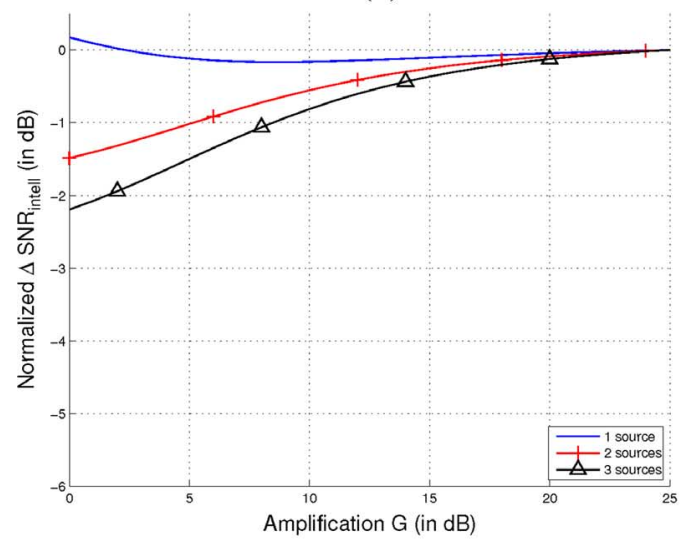

(b)

Fig. 6. Normalized SNR performance of integrated ANC and NR schemes. (a) 2-channel monaural scheme, (b) 4-channel binaural scheme.

error microphone (see also Fig. 5). In this way, for any scenario, the binaural integrated ANC and NR scheme does not have any advantage in terms of causality compared to the monaural integrated ANC and NR scheme. Under a negative causality margin, the ANC would be ineffective and the SNR improvement would merely be due to the binaural NR [9], [14]. For the single noise source scenario, the noise source is at $270^{\circ}$. In the two noise sources scenario, the noise sources are at $270^{\circ}$ and $90^{\circ}$. For the three noise sources scenario, the noise sources are at $270^{\circ}, 90^{\circ}$ and $330^{\circ}$.

The number of noise sources has an impact on the performance of MWF-based NR schemes when signal leakage effects and the effect of the secondary path are neglected, i.e., the performance of the integrated ANC and NR scheme when the gain $G$ is set to a high value [13]. In order to observe the effects of the number of sound sources (speech source plus noise sources) on the ANC part of the integrated ANC and NR schemes it is more convenient to look at the normalized output SNR improvement:

$$
\overline{\Delta \mathrm{SNR}_{\text {intellig }}}=\Delta \mathrm{SNR}_{\text {intellig }}-\Delta \rho_{\text {intellig }}
$$

Where $\Delta \rho_{\text {intellig }}$ is the intelligibility-weighted SNR improvement for the MWF-based NR scheme when no perturbation (signal leakage and secondary path) is taken into account.

Fig. 6 presents the normalized output SNR improvement $\left(\overline{\Delta \mathrm{SNR}_{\text {intellig }}}\right)$ of two integrated ANC and NR schemes (2-channel monaural based on the microphone signals from the left hearing aid and 4-channel binaural), for a gain varying from $0 \mathrm{~dB}$ to $25 \mathrm{~dB}$. For large gains, the integrated $\mathrm{ANC}$ and $\mathrm{NR}$ schemes deliver a normalized output $\mathrm{SNR}\left(\overline{\Delta \mathrm{SNR}_{\text {intell }}}\right)$ of about $0 \mathrm{~dB}$. 
The two integrated ANC and NR schemes are able to deliver an almost constant SNR improvement for any gain when only one noise source is present. When two or more noise sources are present, the normalized SNR performance of the 2-channel integrated NC and NR drops to $-4 \mathrm{~dB}$ to $-5 \mathrm{~dB}$ for low gains. The 4-channel integrated ANC and NR scheme on the other hand allows to maintain a normalized SNR performance above $-2 \mathrm{~dB}$ for up to three noise sources.

\section{CONCLUSION}

It has been shown in previous work that an MWF-based integrated ANC and NR provides an efficient solution to the signal leakage problem in hearing aids with an open fitting. Hearing aids, however, have small dimensions. Therefore, the integrated ANC and NR scheme is subject to strong constraints on causality and on the number of noise sources that can be compensated for.

The binaural MWF-based integrated ANC and NR scheme presented in this paper is based on the microphone signals from both ears. Here, the contra-lateral microphones are distant from the ear canal microphone where the noise is to be cancelled. The propagation time from these microphones to the ear canal microphone is therefore larger and allows, in some scenarios, to design a scheme with a larger causality margin. Therefore, this approach allows to attenuate the noise from a larger number of sources than the monaural integrated ANC and NR scheme.

\section{REFERENCES}

[1] W. Koenig, "Subjective effects in binaural hearing," J. Acoust. Soc. Amer., vol. 22, no. 1, pp. 61-62, 1950.

[2] N. W. MacKeith and R. R. A. Coles, "Binaural advantages in hearing of speech," J. Laryngol. Otol., vol. 85, no. 03, pp. 213-232, 1971.

[3] A. Markides, Binaural Hearing Aids. London, U.K.: Academic, 1977.

[4] P. C. Loizou, Speech Enhancement: Theory and Practice. Boca Raton, FL: CRC, 2007.

[5] L. J. Griffiths and C. W. Jim, "An alternative approach to linearly constrained adaptive beamforming," IEEE Trans. Antennas Propag., vol. AP-30, no. 1, pp. 27-34, Jan. 1982.
[6] J. Chen, J. Benesty, Y. Huang, and S. Doclo, "New insights into the noise reduction Wiener filter," IEEE Trans. Audio, Speech, Lang. Process., vol. 14, no. 4, pp. 1218-1234, Jul. 2006.

[7] S. Doclo, A. Spriet, J. Wouters, and M. Moonen, "Frequency-domain criterion for the speech distortion weighted multichannel wiener filter for robust noise reduction," Elsevier Speech Commun., vol. 49, no. 7-8, pp. 636-656, 2007.

[8] D. P. Welker, J. E. Greenberg, J. G. Desloge, and P. M. Zurek, "Microphone-array hearing aids with binaural output. II. A two-microphone adaptive system," IEEE Trans. Speech Audio Process., vol. 5, no. 6 , pp. 543-551, Aug. 2002.

[9] T. J. Klasen, T. Van den Bogaert, M. Moonen, and J. Wouters, "Binaural noise reduction algorithms for hearing aids that preserve interaural time delay cues," IEEE Trans. Signal Process., vol. 55, no. 4, pp. 1579-1585, Apr. 2007.

[10] S. J. Elliott and P. A. Nelson, Active Control of Sound. Cambridge, U.K.: Academic, 1993.

[11] S. M. Kuo and D. R. Morgan, "Active noise control: A tutorial review," Proc. IEEE, vol. 87, no. 6, pp. 943-973, Jun. 1999.

[12] R. Serizel, M. Moonen, J. Wouters, and S. H. Jensen, "Integrated active noise control and noise reduction in hearing aids," IEEE Trans. Audio, Speech, Lang. Process., vol. 18, no. 6, pp. 1137-1146, Aug. 2010.

[13] R. Serizel, M. Moonen, J. Wouters, and S. H. Jensen, "Output SNR analysis of integrated active noise control and noise reduction in hearing aids under a single speech source," EURASIP Signal Process., vol. 91, no. 8, pp. 1719-1729, Aug. 2011.

[14] B. Cornelis, S. Doclo, T. Van den Bogaert, M. Moonen, and J. Wouters, "Theoretical analysis of binaural multi-microphone noise reduction techniques," IEEE Trans. Audio, Speech, Lang. Process., vol. 18, no. 2, pp. 342-355, Feb. 2010.

[15] X. Kong and S. M. Kuo, "Study of causality constraint on feedforward active noise control systems," IEEE Trans. Circuits Syst. II: Analog Digital Signal Process., vol. 46, no. 2, pp. 183-186, Feb. 1999.

[16] M. Nilsson, S. D. Soli, and A. Sullivan, "Development of the hearing in noise test for the measurement of speech reception thresholds in quiet and in noise," J. Acoust. Soc. Amer., vol. 95, no. 2, pp. 1085-1099, Feb. 1994.

[17] Auditory Tests (Revised), Compact Disc, Auditec,. St. Louis, 1997, Auditec.

[18] J. E. Greenberg, P. M. Peterson, and P. M. Zurek, "Intelligibility-weighted measures of speech-to-interference ratio and speech system performance," J. Acoust. Soc. Amer., vol. 94, no. 5, pp. 3009-3010, Nov. 1993.

[19] A.-S. , "ANSI S3.5-1997 American National Standard Methods for calculation of the speech intelligibility index," Acoust. Soci. of Amer. Jun. 1997, (1997). 